\title{
DE NUEVO CON LOS MIRANDA
}

Los que han estudiado de cerca el episodio del encuentro entre don Quijote y don Diego de Miranda incluyen muchos de los más renombrados críticos de las letras castellanas ${ }^{1}$. Lo que ha generado tanto interés es la yuxtaposición de los dos y el gran contraste de sus modi vivendi, además de la gran victoria que da lugar al título de Caballero de los Leones. En resumen parece que existen un «buen don Diego" y un "mal don Diego» y toda una variedad de opiniones sobre cómo el uno y el otro impactan nuestro entendimiento del propósito artístico de Cervantes al crear el encuentro. Lo que propongo yo me aleja de este debate, pues pienso concentrarme en los vaivenes anímicos o sea sentimentales de don Quijote, su tesitura íntima, cuando acompaña a los Miranda dentro y fuera de aquella casa «que semejaba un monasterio de cartujos» ${ }^{2}$. Creo que es posible que Cervantes hubiera querido indicarnos que la imagen de la Dulcinea encantada nunca está muy lejos de los pensamientos y emociones de este leal amante; aquella preocupación no tan sólo tiende a anular el orgullo de haber salido victorioso en una batalla que ha probado la realidad de su caballería, sino que tam-

\footnotetext{
1 BRYANT L. CREel hace un breve resumen de las opiniones conflictivas en Don Quijote, Symbol of a Culture in Crisis. Ediciones Hispanófila 47. Valencia, Albatros, 1988, pp. 53-57. Más intenso es el acabado estudio de CHARLEs D. PRESBERG, "Yo sé quién soy': Don Quixote, Don Diego de Miranda and the Paradox of SelfKnowledge», Cervantes. Bulletin of the Cervantes Society of America XIV, 2, 1994 pp. 41-69.

2 Miguel De Cervantes SaAvedra, Don Quijote de la Mancha, II, 18. De aquí en adelante señalo parte y capítulo en paréntesis, dejando que el lector emplee su preferida edición; las citas que no se identifican se encuentran en el mismo capítulo que la anterior.
} 
bién sirve de fondo grisáceo tanto para el dorado éxito de la confrontación con los leones como para la argentada rendición del Caballero de los Espejos.

Al llegar al capítulo XV de la segunda parte del Quijote acabamos de presenciar la victoria del protagonista sobre el que no fue ni podía ser quien parecía: «'aunque parecéis el bachiller Sansón Carrasco, no lo sois, sino otro que le parece, y que en su figura aquí me le han puesto mis enemigos, para que detenga y temple el ímpetu de mi cólera, y para que use blandamente de la gloria del vencimiento"» (II, 14). Tal gloria deja al andante "[e]n estremo contento, ufano y vanaglorioso», primeras palabras del capítulo XV en un párrafo que añade otra referencia al efecto negativo de aquellos inímicos: «el encantamiento de su señora pasaba adelante». Yuxtaponer estas dos referencias en tan breve espacio no será fortuito.

Si saltamos lo demás del capítulo, es decir, pasando por alto la conversación entre Sansón y el igualmente transformado Tomé Cecial, así para seguir de cerca la carrera del protagonista, Cervantes nos repite lo del enaltecimiento: «Con la alegría, contento y ufanidad que se ha dicho seguía don Quijote su jornada», primeras palabras del capítulo XVI, seguidas unos renglones después por esto: «tenía en poco a los encantos y a los encantadores»; otra vez la colocación parece consciente. A pesar de esta supuesta confianza en sí, don Quijote luego ha de defender ante las dudas de Sancho la explicación de un no-Sansón, un no-Tomé, recurriendo a un tema favorito pero uno que en este momento contradice en algo la denigración de la eficacia de los encantadores: "Todo es artificio y traza [...] de los malignos magos que me persiguen'». Luego explica lo que hicieron para que el vencido se pareciera al bachiller, lo mismo que con su dama: "no ha dos días que viste por tus mismos ojos la hermosura y gallardía de la sin par Dulcinea en toda su entereza y natural conformidad, y yo la vi en la fealdad y bajeza de una zafia labradora, con cataratas en los ojos y con mal olor en la boca $\left.[\ldots]^{\prime}\right)^{3}$. Declara sin vacilación que el "'perverso encantador"» que efectuó tal desfiguración, "no es mucho que haya hecho"» lo mismo con Carrasco y Cecial. Sancho, no convencido sino de su propia industria, responde, creo que lacónica si no evasivamente, "'Dios sabe la verdad de todo'».

Enseguida entra en escena el que llevará el título descriptivo de «el Caballero del Verde Gabán» ${ }^{4}$. Los dos caballeros se miran y ad-

3 Nótese que don Quijote defiende su fantasía corrigiendo con cataratas la frase de Sancho, "las perlas de los ojos'»(II, 10), la cual había criticado en el capítulo 11 .

4 PrEsberg, op. cit., 42, n. 3: "It is worth noting that Don Quixote is never quoted as using this sobriquet for Don Diego», pero la distinción es central en el 
miran, y don Quijote rompe el silencio refiriéndose a su triste figura. Describe su misión caballeresca y se jacta de su fehaciente historia: "he merecido andar ya en estampa en casi todas o las más naciones del mundo'». Es claro que continúa con su ufanidad, y aún aumentada, sin duda para impresionar, pero sorprende que el andante describe su propia figura, de veras triste y "'tan nueva y tan fuera de las que comúnmente se usan'», palabras suyas en una fina combinación de realidad y fantasía, cordura y locura, la misma oscilación que se repetirá durante toda su visita con los Miranda: «así que, señor gentilhombre, ni este caballo, esta lanza, ni este escudo ni escudero, ni todas juntas estas armas, ni la amarillez de mi rostro, ni mi atenuada flaqueza, os podrá admirar de aquí adelante, habiendo ya sabido quién soy y la profesión que hago'» ${ }^{5}$. Llamarle al otro «gentilhombre», título más cortesano que caballeresco, ¿`será un anticipo de su conocido desprecio directo?

Pero algo falta en esta escena: don Qujote no menciona a Dulcinea a quien acaba de defender contra el embuste del Caballero de los Espejos: "de lo que yo más me precio y ufano es de haber vencido en singular batalla a aquel tan famoso caballero don Quijote de la Mancha, y héchole confesar que es más hermosa mi Casildea que su Dulcinea'»(II, 14). ¿Qué ha pasado con el deber de ser amante, un deber que él mismo ha ido reconociendo desde el principio: «no le faltaba otra cosa que buscar una dama de quien enamorarse; porque el caballero andante sin amores era árbol sin hojas y sin fruto y cuerpo sin alma» (I, 1). No, en este momento don Quijote se ofrece, para decirlo así, deshojado, infructuoso, desanimado, aunque sí, riguroso en sus declaraciones; o su fantasía no admitirá la sustitución de "una aldeana soez y baja'», frase suya (II, 14), o conscientemente opta por no introducir un tema que le ha ido provocando sofisterías a lo largo de su carrera.

El del Verde Gabán, después de dudar que haya un andante verdadero que pueda "'poner en olvido las innumerables [historias] de los fingidos caballeros andantes'»(II, 16), responde cortésmente describiendo su propia vida, y de ello brota el contraste que ha ocasionado tantas discusiones además de la consabida reacción de Sancho: " "me parece vuesa merced el primer santo a la jineta que he visto en todos los días de mi vida'». Recordemos, sin embargo,

\footnotetext{
episodio: «after carefully scrutinizing Don Diego, Don Quixote considers that other character to follow a very different 'profession' from his own, one which the protagonist fails to specify - probably because the profession of a more than moderately wealthy, rural hidalgo falls outside his chivalric schemata", aunque de su uso del título gentilhombre (véase abajo) será posible inferir su opinión.

5 La realidad se confirma una vez en la casa, en la descripción de sus atavíos y la tentativa de limpiarse con un indeterminado número de cubos de agua.
} 
esta frase de Randolph D. Pope: "Son sospechosas la elogiosa descripción que don Diego hace de sí mismo, así como su vestimenta, que a pesar de ceñirse en todo a la moda del momento, incluso con moderación, no deja de revelar cierta vanidad y sugiere, por el predominio del verde - color asociado a lo erótico y a los bufonesalguna extravagancia» ${ }^{6}$.

Cuatro renglones en adelante ha de asustarnos la alusión a la "profunda malencolía» de don Quijote que ha sido disuelta por haberse reído de la declaración de Sancho. ¿De dónde ha brotado tan repentinamente esta melancolía? ¿Por envidiar la vida de este gentilhombre, una vida cómoda que no dista mucho de ser lo más opuesta de la caballeresca suya? Es posible, pero para mí la causa es la expresa incredulidad del caballero de «la hermosa yegua tordilla» quien ante la razonada explicación y la evidencia física encarnada en don Quijote mismo, responde diciendo "No me puedo persuadir que haya hoy en la tierra quien favorezca viudas, ampare doncellas, ni honre casadas, ni socorra huérfanos, y no lo creyera si en vuesa merced no lo hubiera visto con mis ojos' $\gg{ }^{7}$. Esta afirmación, es posible sea irónica, pero en las últimas palabras creo ver el escondido motivo de la confrontación con el león en su jaula: don Quijote necesitará probar con creces su tan nueva vocación; obras, en vez de palabras. También de ello nacerá más tarde aquella despectivísima declaración de gran resonancia: «'Váyase vuesa merced, señor hidalgo, [...] a entender con su perdigón manso y con su hurón atrevido, y deje a cada uno hacer su oficio'» (II, 17) ${ }^{8}$.

Al fin y al cabo, el andante consigue una victoria que celebrará con el nuevo apodo de "El Caballero de los Leones», y si el vencimiento del Caballero de los Espejos fue, como dice Eduardo Urbina, «una afortunada victoria que encaja perfectamente con las necesidades presentes de DQ y los requisitos futuros de su ficción" ${ }^{9}$, no lo es menos esta victoria frente al león. Y para hacer aún más clara la distinción entre las dos carreras don Quijote vuelve al tema

6 Lectura del capítulo XVI de la segunda parte de Miguel DE Cervantes SAAVEDRA, Don Quijote de la Mancha, edición del Instituto Cervantes dirigida por Francisco Rico, II. Barcelona, Instituto Cervantes / Crítica, 1998, p. 139.

7 ¿Existirá la posibilidad de que minan su confianza e incrementen la melancolía las dos victorias menos que cien por cien satisfactorias? Intervinieron los encantadores en la una complicando el carácter del logro, en la otra el león le desdeñó con el mismo resultado, razón posible de pedir un testimonio escrito. Empero la insistencia textual en la ufanidad de don Quijote, creo que anula tal hipótesis porque tanto contentamiento equivale a aceptar los hechos según su propia perspectiva y no la nuestra.

8 ¿Cómo leer en alta voz la frase señor hidalgo?

9 Lectura del capítulo XV de la segunda parte de Don Quijote, ed. Instituto Cervantes, II, pp. 138-39. 
de andantes y cortesanos; se destaca el andante, por supuesto, quien en el despoblado "'anda buscando peligrosas aventuras, con intención de darles dichosa y bien afortunada cima, sólo por alcanzar gloriosa fama y duradera'». Dicho y hecho, en efecto: deber cumplido, y no hace falta un hurón atrevido. Joaquín Casalduero nota que don Quijote «defiende sus actos con una serenidad en la cual resplandece el brío de todas sus victorias» ${ }^{10}$. También, creo yo, con la satisfacción (por momentánea que sea) de que salió bien la confirmación de su oficio: "porque mejor suena en las orejas de los que lo oyen "el tal caballero es temerario y atrevido" que no "el tal caballero es tímido y cobarde"». Estas palabras podrían ser una clara afrenta al honor de don Diego de Miranda pero él parece no entenderlas como tales, antes acepta el razonamiento de don Quijote y muy cortésmente le invita a descansar en su casa.

Es curioso que nuestro héroe no pidiera al leonero que pasase por El Toboso para blasonar su victoria, sino que se contentara con un testimonio escrito ${ }^{11}$ sin mencionar la demanda que hizo a los galeotes: "es mi voluntad, que [...] luego os pongáis en camino y vais a la ciudad del Toboso, y allí os presentéis ante la señora Dulcinea del Toboso'» (I, 22) para que ella sepa y reconozca su valor y valentía. Pero en aquel momento la soñada realidad de su dama sin par todavía quedaba firmemente en pie. En cambio, al iniciar su tercera salida don Quijote mismo buscó a aquella princesa de La Mancha; desafortunado y desorientado por no topar con aquel palacio, queda un "ejemplo de desdichados, [...] blanco y terrero donde tomen la mira y asiesten las flechas de la mala fortuna'» (II, 10). Cuando Sancho le presenta la ruda y apestosa transformada, quedan eclipsado el sol de su alma e iniciadas sus hondas preocupaciones.

Entendamos lo dicho y lo no dicho en las palabras de don Quijote al referirse a lo que acaba de hacer con el león: «'No debo más, y encantos afuera, y Dios ayude a la razón y a la verdad, y a la verdadera caballería, y cierra, como he dicho, en tanto que hago señas a los huídos y ausentes, para que sepan de tu boca esta hazaña"» (II, 17). De las frases "verdadera caballería'» y "huídos y ausentes'” se infiere la misma oposición que se ha presentado desde el principio en el contraste entre los dos caballeros, o sea entre el caballero por antonomasia y el gentilhombre en su yegua. Pero la frase "encantos afuera'», parece ser un tácito recuerdo del encantamiento de Dulcinea ${ }^{12}$. Da la seña a los ausentes, «todos en tropa

10 Sentido y forma del Quijote (1605-1615). Madrid, Ínsula, 1966, p. 260.

11 Valga la pregunta: ¿para un apéndice en el segundo tomo de su historia?

12 ¿Es don Quijote consciente de la mentira de Sancho pero quiere evitar las consecuencias de admitírsela? 
y antecogidos del hidalgo" y escuchan todos la narrativa debidamente elogiosa de parte del leonero que da lugar a la pregunta de don Quijote "Qué te parece desto, Sancho? [...] ¿Hay encantos que valgan contra la verdadera valentía?’» Los encantadores le quitaron la ventura, dice, "pero el esfuerzo y el ánimo, será imposible’. Aún en esta segunda gran victoria descubrimos la preocupación subyacente por el encantamiento de Dulcinea.

Luis Murillo opina que en su igual social don Quijote percibe un reto moral, en contraste con el reto caballeresco del Caballero de los Espejos ${ }^{13}$. No veo un choque aquí en el campo de la moralidad sino otro en el de la caballería misma. Don Diego se ufana, en efecto, de su vida ejemplar pero es una vida contemplativa, rutinaria, y sus buenas obras - de las cuales hace alarde diciendo no alardear- parecen más bien por su deber social de hacendado que no por sentir un verdadero impulso caritativo. Y este padre prestigioso que se siente decepcionado porque su único hijo no cumple con el deseo burgués paternal de que se prepare para una carrera legítima, es difícil pensar de él como vástago de los adalides de Castilla la Nueva ${ }^{14}$.

13 A Critical Introduction to Don Quixote. New York, Peter Lang, 1988, p. 151.

14 Se puede pensar en él como el Caballero de la Aburrida Prudencia, influenciado por PRESBERG, op. cit., pp. 47-48: «in the three chapters devoted to Don Diego, that temperate gentleman seems to exemplify the most important cardinal virtue of prudence or discreción, always and everywhere the quintessence of the golden mean». José ANTONIO MARAVALL le califica un "tan juicioso y prudente hidalgo castellano" además de "aldeano hidalgo» (Utopía y contrautopía en el Quijote. Santiago de Compostela, Pico Sacro, 1976, pp. 160 y 185). JosÉ GARCía GIBERT emplea la frase "campeón de la sensatez" — ¿irónicamente?- en Cervantes y la Melancolía. Ensayos sobre el tono y la actitud cervantinos, Novatores 7. Valencia, Edicions Alfons el Magnànim y Generalitat Valenciana, 1997, p. 30. Para FrANCISco Márouez Villanueva don Diego es un «orate de la vida prudente y dosificada con la que se imagina esquivar los riesgos que a todo hombre acechan en la tarea de vivir» ("La locura emblemática en la segunda parte del Quijote», Cervantes and the Renaissance, ed. Michael D. McGaha. Easton, Juan de la Cuesta, 1980, p. 92). ROBERT W. FELKEL reconoce la pobreza novelística de don Diego: «La misma perfección [sic] de don Diego de Miranda impide que sea lo suficientemente interesante como para ser el protagonista de una novela en el sentido moderno de la palabra. Resulta un poco incoloro, a pesar de su indumentaria» («Una cuestión ética en Cervantes: Menosprecio de defecto y alabanza de exceso en el Quijote», Anuario de Letras. Revista de la Facultad de Filosofía y Letras [Universidad Autónoma de México], XXVI, 1988, 117-36). Gerald L. Gingras defiende el modo de vestir en «Diego de Miranda: Bufón or Spanish Gentleman?», Cervantes. Bulletin of the Cervantes Society of America, V, 2, 1985, pp. 129-140. HENRY W. SUlLIVAN propone el sueño de don Quijote en la Cueva de Montesinos un anticipo del desdoblamiento inherente en la yuxtaposición Quijote-Miranda, "the experience of splitting of reduplication [...] that is typical of dreaming. Both the Knight of the Mirrors, with his bogus squire and 'reflected' image usurped from Don Quixote, and Don Diego de Miranda (the healthy country gentleman that Don Quixote might have been, 
Las tinajas. ¿Por qué éstas tan fácilmente hacen que don Quijote tan sólo verlas piense inmediatamente en «su encantada y transformada Dulcinea» (II, 18) cuando no la mencionó poco antes al describir su oficio? ${ }^{15}$. ¿Por qué en el acto de verlas cita el décimo soneto de Garcilaso, versos virgilianos que presentan, en la frase de Ángel Prieto de Paula, «la emocionada evocación de la amada muerta» del poeta -repito, «muerta»? ${ }^{16}$. ¿Cómo debemos entender la siguiente exclamación: «¡Oh tobosescas tinajas, que me habéis traído a la memoria la dulce prenda de mi mayor amargura'»? En esta sucinta expresión del agridulce choque entre lo que fantasea don Quijote y la realidad mentirosamente contrastada por Sancho vemos el momento más obvio de la continua inquietud íntima del protagonista.

En efecto ni la victoria sobre el transformado Sansón Carrasco, ni la negativa a la coincidencia entre la cara de su escudero y la de Tomé Cecial, ni la pensada defensa de su oficio, han borrado de la memoria de don Quijote la preocupación por su mala fortuna, una preocupación que parece instar a que el amante andante alabe hasta los cielos la glosa de don Lorenzo porque raya en el mismo tema de algo valioso perdido y las esperanzas de su reaparición ${ }^{17}$ :

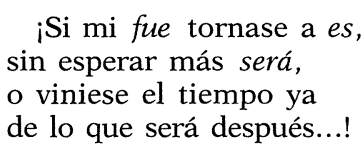

O sea, «ojalá que mi Dulcinea original volviera cuanto antes para quitarme las penas y ansiedades que me siguen molestando». Una estrofa más de don Lorenzo repite la idea:

function like Doppelgänger to the hero" (Grotesque Purgatory. University Park, The Pennsylvania State University Press, 1996, p. 127; su título nos lleva a meditar las ironías del caso Miranda).

15 Hasta este momento en el episodio con el del Verde Gabán ha citado el nombre de su amada dos veces, al conversar con Sancho y al encomendarse a ella estando solo.

16 Garcilaso de la Vega, Poesías completas, Castalia Didáctica 21, 2. ${ }^{\mathrm{a}}$ ed. Madrid, Castalia, 1989 , p. 56, n. 8. Lo que Prieto escribe a continuación también ofrecerá alguna relevancia al caso de don Quijote y a la glosa de don Lorenzo: «El soneto señala los pasos de la historia: serenidad dolorida anterior [...]; evocación del pasado feliz desde la ausencia definitiva; deseo de superar esa situación morbosa; choque con el presente, que se contempla como un mar de recuerdos en el que el poeta naufraga» (cursivas mías).

17 Dominick Finello ha escrito que la opinión de la poesía que les ofrece don Quijote a los Miranda es uno de sus discursos inspirados por aquel género que brotan de "a mind that is intense and self-assured» (Pastoral Themes and Forms in Cervantes's Fiction. Lewisburg, Bucknell University Press, 1994, pp. 103-104, cursiva mía); creo yo que este elogio es distinto de esos dictámenes generales porque se derivan fundamentalmente de la dudosa confianza en sí mismo a raíz del encantamiento de Dulcinea. 


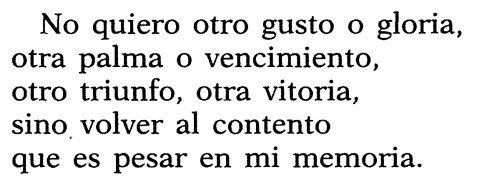

Los elogios hiperbólicos de don Quijote ${ }^{18}$, además de alabar al verdadero poeta, insisten en que los lectores nos fijemos bien en esta glosa para descubrir su tema.

Este primer soneto efectivamente concluye la interacción significativa de don Quijote con los Miranda. El soneto que sigue es, según Giuseppe Grilli, «el elemento literario central del capítulo [y] asimismo una representación interesante de la técnica de digresión narrativa tantas veces encontrada en el Quijote» (subrayado mío). Por lo tanto prefigura las bodas de Camacho y el triunfo de Basilio y poco tiene que ver con el tema de Dulcinea; aunque dos versos hacen eco de la lucha de nuestro héroe - "'amor suele de hecho / facilitar la más difícil cosa'»- el poema saca sólo un breve comentario de don Quijote y en alabanza de su artificio. El párrafo siguiente anuncia la salida del caballero y escudero después de sus cuatro días de ocio.

En resumen, por lo que dice don Quijote, y por lo que no dice, creo que ni la victoria de desarzonar al Caballero de los Espejos, ni la de esperar valientemente la embestida de un enorme león africano, ni la satisfacción de distinguirse como caballero andante ante quien es, al parecer de don Quijote, un yegüero pusilánime, nada de esto basta para borrar del todo la aniquilada imagen de la reina de sus pensamientos. Con buena razón ha escrito Javier García Gibert que «Ese punto de intersección en el que confluyen las cosas buenas, que originan la alegría, y las malas, que provocan la tristeza, es justamente el estado de ánimo 'entre triste y alegre' que la literatura de Cervantes refleja con tanta fidelidad y perseverancia» ${ }^{19}$. A pesar de haber confirmado su valentía andantesca, don Quijote no ha podido lograr el contentamiento de una plena realización de su carrera vital porque sigue molestándole en su intimidad la imagen trastrocada de su adorada.

ROBERT L. HATHAWAY

Colgate University

\footnotetext{
18 "A propósito del Quijote, II, 18", Actas del II Coloquio Internacional de la Asociación de Cervantistas. Alcalá de Henares, 6-9 noviembre 1989. Barcelona, Anthropos, 1991, p. 490.

19 Op. cit., p. 14.
} 


\section{BIBLIOGRAFÍA}

Casalduero, Joaduín, Sentido y forma del Quijote (1605-1615). Madrid, Ínsula, 1966. Cervantes SaAvedra, Miguel De, Don Quijote de la Mancha, ed. Martín de Riquer. Barcelona, Juventud, 1958.

- Don Quijote de la Mancha, ed. Instituto Cervantes dirigida por Francisco Rico. Barcelona, Instituto Cervantino / Crítica, 1998.

Creel, Bryant L., Don Quijote, Symbol of a Culture in Crisis, Ediciones Hispanófila 47. Valencia, Albatros, 1988.

Finello, Dominick, Pastoral Themes and Forms in Cervantes's Fiction. Lewisburg, Bucknell University Press, 1994.

García Gibert, JAVIER, Cervantes y la Melancolía. Ensayos sobre el tono y la actitud cervantinos, Novatores 7. Valencia, Edicions Alfons el Magnánim /Generalitat Valenciana, 1997.

Garcilaso de la Vega, Poesías completas, ed. Ángel Prieto de Paula. Castalia Didáctica 21, 2. ${ }^{a}$ ed. Madrid, Castalia, 1989.

GRILLI, GIUSEPPE, "A propósito del Quijote, II, 18», Actas del II Coloquio Internacional de la Asociación de Cervantistas. Alcalá de Henares, 6-9 noviembre 1989. Barcelona, Anthropos, 1991, pp. 485-491.

Maravall, José AnTonio, Utopía y contrautopía en el Quijote. Santiago de Compostela, Sacro Pico, 1976.

Márouez VillanueVa, Francisco, "La locura emblemática en la segunda parte del Quijote», Cervantes and the Renaissance, ed. Michael D. McGaha. Easton, Juan de la Cuesta, 1980, pp. 87-112.

MuRIllo, LuIS, A Critical Introduction to Don Quixote. New York, Peter Lang, 1988. Presberg, Charles D., "'Yo sé quién soy': Don Quixote, Don Diego de Miranda and the Paradox of Self-Knowledge», Cervantes. The Bulletin of the Cervantes Society of America, XIV, 2, 41-69.

Sullivan, Henry W., Grotesque Purgatory. A Study of Cervantes's Don Quixote, Part II. University Park: The Pennsylvania State University Press, 1996. 